\title{
Identification of Schistosoma mansoni candidate antigens for diagnosis of schistosomiasis
}

\author{
Gardenia Braz Figueiredo Carvalho', Rosiane Aparecida da Silva-Pereira², \\ Lucila Grossi Gonçalves Pacífico', Cristina Toscano Fonseca ${ }^{1,3 /+}$ \\ ${ }^{1}$ Laboratório de Esquistossomose 2Laboratório de Parasitologia Celular e Molecular, \\ Instituto de Pesquisas René Rachou-Fiocruz, Av. Augusto de Lima 1715, 30190-002 Belo Horizonte, MG, Brasil \\ ${ }^{3}$ Instituto Nacional de Ciências e Tecnologia em Doenças Tropicais
}

\begin{abstract}
The development of a more sensitive diagnostic test for schistosomiasis is needed to overcome the limitations of the use of stool examination in low endemic areas. Using parasite antigens in enzyme linked immunosorbent assay is a promising strategy, however a more rational selection of parasite antigens is necessary. In this study we performed in silico analysis of the Schistosoma mansoni genome, using SchistoDB database and bioinformatic tools for screening immunogenic antigens. Based on evidence of expression in all parasite life stage within the definitive host, extracellular or plasmatic membrane localization, low similarity to human and other helminthic proteins and presence of predicted B cell epitopes, six candidates were selected: a glycosylphosphatidylinositol-anchored $200 \mathrm{kDa}$ protein, two putative cytochrome oxidase subunits, two expressed proteins and one hypothetical protein. The recognition in unidimensional and bidimensional Western blot of protein with similar molecular weight and isoelectric point to the selected antigens by sera from $\mathrm{S}$. mansoni infected mice indicate a good correlation between these two approaches in selecting immunogenic proteins.
\end{abstract}

Key words: Schistosoma mansoni - diagnosis - antigen candidate - bioinformatics analysis

Recently, in Brazil an increased number of low schistosome endemic areas, expanded geographical extension of the disease, appearance of new foci and acute outbreaks of the disease have been observed, indicating that although control programs succeeded on reducing morbidity and mortality they failed to control the disease transmission (Katz 1998, Barbosa et al. 2001, Enk et al. 2003). The lack of effective diagnostic assays to identify individuals with low parasite burden is one factor that contributes to transmission (Enk et al. 2008).

Currently, the common used method in the diagnostic of schistosomiasis is stool examination, using the Kato-Katz method. However, this method presents limitation in the detection of positive individuals, when the intensity of infection is low and it is less effective in determining the prevalence in low endemic areas (Feldmeier \& Poggensee 1993, Kongs et al. 2001).

The detection of antibodies specific to parasite antigens is more sensitive to detect light infections and can be applicable to large scale examination (Sorgho et al. 2005, Alarcón de Noya et al. 2007). The enzyme linked immunosorbent assay (ELISA) is a serological test useful for epidemiological studies, due to its high sensitivity for the diagnosis of schistosomiasis, de-

Financial support: RIPAG/FIOCRUZ-MG, PAPES V/FIOCRUZ, CPqRR/FIOCRUZ-MS, FAPEMIG

Fellowships: FAPEMIG (to GBFC), PV/CNPq (to LGGP), PQ/ $\mathrm{CNPq}$ (to CTF).

+ Corresponding author: ctoscano@cpqrr.fiocruz.br

Received 20 April 2011

Accepted 26 July 2011 pending on the Schistosoma mansoni antigen used in the test (Ishida et al. 2003, Sorgho et al. 2005, Alarcón de Noya et al. 2007, Luo et al. 2009), and the possibility for automating the process. However, until now the utilization of the ELISA for diagnosis of schistosomiasis does not discriminate ongoing and previous infection (KatoHayashi et al. 2010). In addition, some antigens used in ELISA present cross reaction with antigens from other helminths as ancylostomides and Ascaris lumbricoides (Correa-Oliveira et al. 1988, Ishida et al. 2003, Luo et al. 2009). A rational selection of antigens to be used in the serological diagnosis of schistosomiasis may overcome this ELISA limitation use.

The availability of complete genome sequences in combination with novel advanced technologies have contributed to vaccinology field, leading to vaccine development starting from the prediction of all antigens in silico, independently of their abundance and without the need to grow the pathogen in vitro, a strategy known as reverse vaccinology (Capecchi et al. 2004, Bai \& Borrow 2010, Zhao et al. 2011).

In this context, following the same principles of the reverse vaccinology, it would be possible using bioinformatics tools and performing in silico analysis to select promising antigens to be used for diagnosis of the schistosomiasis mansoni, since the $S$. mansoni genome had already been sequenced (Berriman et al. 2009).

The important features to identify promising antigens for immunodiagnosis of schistosomiasis must be based on: expression of the gene encoding the protein in all the three different stages of the parasite's life cycle within the definitive host, the localization of antigens in the parasite (secreted or on the parasite surface), low similarity to human and other helminthic proteins and presence of predicted B cell epitopes. 
In the present study we have proposed a new strategy to identify antigen candidates to be used in the schistosomiasis diagnosis based on the detection of specific antibodies against these antigens by ELISA.

\section{MATERIALS AND METHODS}

In silico analysis - Evidence of gene expression - To select schistosome proteins with evidence of expression in the three parasite life cycle stages on the definitive host (schistosomulum or lung schistossomulum, adult worm and egg) the SchistoDB database (schistodb.net/schistodb20/) was accessed. The SchistoDB contains several different $S$. mansoni data sets and the results of different computational analyses are integrated in a relational database that has permitted us to apply a technique known as genomic filtering. The current 2.0 database version provides access to the latest draft of $S$. mansoni genome sequence and annotation obtained from the Wellcome Trust Sanger Institute and the mitochondrial genome.

Cellular localization - To select proteins that are expressed on the parasite surface or secreted by the parasite we performed in silico analysis for the presence of signal peptide, transmembrane domain and topology and also the prediction of cellular localization. The genes encoding proteins with evidence for gene expression in the three stages of the parasite life cycle on the definitive host were first submitted to signal peptide prediction using the software Server SignalP 3.0 (cbs.dtu.dk/services/ SignalP/) (Expasy Proteomics Server). The prediction of transmembrane domain was performed using the software SOSUI Engine version 1.11 (bp.nuap.nagoya-u.ac.jp/ sosui/) (Expasy Proteomics Server) that predicts a part of the secondary structure of proteins from a given amino acid sequence. The cellular localization was predicted using the SherLoc software (bs.informatik.uni-tuebingen. de/Services/SherLoc/) (Applied Bioinformatics Group) that is a new comprehensive system for predicting the localization of eukaryotic proteins.

Human and helminths similarity - To access the similarity between selected proteins in the previous in silico analysis with human or helminthic proteins, the amino acid sequences were compared with the National Center for Biotechnology Information protein database using the BlastP algorithm (blast.ncbi.nlm.nih. gov/Blast.cgi) (BLAST - Basic Local Alignment Search Tool) using the default parameters of this algorithm.

$B$ cell epitope prediction - The B cell epitope prediction was performed using the program BCPREDS B-cell epitope prediction Server (ailab.cs.iastate.edu/ bcpreds/) (Artificial Intelligence Research Laboratory) available online. The software for the detection of linear B cell epitopes is based on the Support Vector Machines (SVM) - machine learning method using subsequence kernel (SSK) in building SVM classifiers.

Characterization of the selected proteins - To characterize the selected proteins we performed the prediction of molecular weight and isoelectric point using the software Compute pI/Mw tool (expasy.org/tools/pi_tool. html) (Expasy Proteomics Server) and the prediction of potential O-glycosylation sites using the OGPET v 1.0 (ogpet.utep.edu/OGPET/) (Biological Sciences).

Antigens preparation - Cercariae obtained from Biomphalaria glabrata snails exposed to light for $2 \mathrm{~h}$ were mechanically transformed in skin-stage schistosomula according to Ramalho-Pinto et al. (1974). Briefly, cercariae were incubated on ice for $30 \mathrm{~min}$ and centrifuged for $3 \mathrm{~min}, 1.800 \mathrm{~g}, 4^{\circ} \mathrm{C}$. The cercariae were resuspended in $1 \mathrm{~mL}$ of cold Earle's salts plus Earle's lactalbumin hydrolysate (ELAC) containing $0.5 \%$ lacto albumin, $1 \%$ penicillin/streptomycin and $0.17 \%$ glucose. The tails were detached by vortex in high speed for $2 \mathrm{~min}$. Afterwards the tails were removed by washing with ELAC for six times. The schistosomula were incubated for $1 \mathrm{~h}$ and $30 \mathrm{~min}$ at $37^{\circ} \mathrm{C}$ in ELAC and washed with apyrogenic physiologic saline. The adult worms (male and female LE strain $S$. mansoni) were obtained from infected mice. The schistosomula antigens preparation (SAP) and adult worm antigens preparation (AWP) were obtained by homogenization of recently transformed schistosomula and adult worms, respectively, in 37 cycles of $30 \mathrm{~s}$ on ice using virtz, with 1 min interval on ice between each cycle. After homogenization, antigen preparations were submitted to centrifugation at $340 \mathrm{~g}$ for $1 \mathrm{~h}$ at $4^{\circ} \mathrm{C}$. Antigen preparations were dialysed against physiologic saline $0.85 \%$. The total protein concentration content of each preparation was determined according to Bradford (1976).

Western blot unidimensional (1D) - AWP and SAP proteins were separated on a $10 \%$ or $12 \%$ denaturing polyacrylamide gel by electrophoresis (Laemmli 1970). From the gel, the protein was transferred to nitrocellulose membranes (Amersham Biosciences) as previously described by Towbin et al. (1979). The nitrocellulose membranes were blocked with $5 \%$ dry milk at $4^{\circ} \mathrm{C}$ overnight and reacted with pool of serum from infected mice diluted $1: 40$ for $1 \mathrm{~h}$ at room temperature (RT). After three washes with $0.5 \mathrm{M} \mathrm{NaCl}-0.02 \mathrm{M}$ Tris-buffered saline $(\mathrm{pH}$ 7.5 ), $0.05 \%$ Tween 20 (TBST), the membranes were incubated for $1 \mathrm{~h}$ at RT with anti-mouse IgG-alkaline phosphatase conjugate 1:5.000 (SouthernBiotec) in TBST plus $3 \%$ dry milk. After three washes with TBST, the membranes were treated with alkaline phosphatase reaction developing buffer together with the BioRad revelator kit.

Western blot bidimensional (2D) - A hundred micrograms of AWP were loaded on immobilized $\mathrm{pH}$ gradient strip with linear gradient separation of $\mathrm{pH}$ ranging from three-10 (Bio-Rad). The strip was submitted to rehydration and isoelectric focalization at $20^{\circ} \mathrm{C}$ and $50 \mathrm{~mA} /$ strip in Protean IEF Cell (Bio-Rad). Passive rehydration was performed for $4 \mathrm{~h}$ followed by an active rehydration at $50 \mathrm{~V}$ for $12 \mathrm{~h}$. Isoelectric focusing was gradually increased to $4.000 \mathrm{~V}$ and run for $16.000 \mathrm{~V}$-h. The strip was equilibrated in a buffer containing dithiothreitol and after iodoacetamide. The molecular weight standard (Pre-stained BenchMark, Invitrogen) and the strip were placed on $15 \%$ sodium dodecyl sulfate polyacrylamide gel electrophoresis (SDS-PAGE) and sealed with $0.5 \%$ agarose solution. The electrophoresis was carried out 
under $100 \mathrm{~V}$ constant voltage. From the gel, the proteins were transferred to polyvinylidene difluoride (PVDF) membranes (Amersham Biosciences) as previously described by Towbin et al. (1979). The PVDF membranes were blocked with 3\% dry milk, then washed twice for 30 min with TBST and incubated with serum pool from infected mice (1:40) overnight. After washes with TBST, the second antibody, an anti-mouse IgG conjugated to peroxidase diluted 1:5.000, was added to the membrane and incubated at RT for $2 \mathrm{~h}$. For detection, the membrane was incubated with diaminobenzidinetetrahydrochloride peroxidase substrate solution (Sigma Aldrich).

Ethics - All procedures involving animals were approved by the local Ethical Commission on Animal Use from Fiocruz (LW25-10).

\section{RESULTS}

In silico analysis - In order to select $S$. mansoni antigen candidates for the immunodiagnosis of schistosomiasis, in silico analyses were performed using a variety of bioinformatics tools. In the first analysis in SchistoDB database, based on evidence of gene expression at different stages of the parasite's life cycle (lung schistosomula or schistosomula, adult worms and eggs) or based on the presence of signal peptide, 21.168 sequences were found and many were redundant sequences, since the genome has nearly 13.000 transcripts (Berriman et al. 2009). By crossing these data and select only those sequences that were common to all life stages of the parasite on the definitive host and also presented a predicted signal peptide, this number was reduced to 72 sequences (Fig. 1).

Subsequently, these 72 sequences were submitted to three more in silico analyses. We first performed the prediction of transmembrane domain using the software SOSUI, where 59 out of the 72 protein sequences had at least one predicted transmembrane domain (data not shown). Next, we performed the prediction of cellular localization in SherLoc in order to see if the sequences were secreted or exposed. From the 72 sequences analyzed, 20 were predicted as transmembrane proteins and 16 were predicted as secreted proteins. The other proteins were predicted to be peroxisomal, golgi apparatus, endoplasmatic reticulous and lysosomal proteins (data not shown). We also performed a search for similarity in a protein database using the BlastP algorithm to determine the percentage of similarity of the 72 selected sequences with human proteins and with proteins from other helminths. None of them had similarity with any helminthic proteins. Regarding human proteins, 55 sequences had similarity with human proteins ranging from $9-99 \%$ of similarity (data not shown). The proteins that presented at least $60 \%$ similarity with human proteins were excluded from our analysis as well as those proteins predicted to be peroxisomal, golgi apparatus, endoplasmatic reticulous and lysosomal proteins. After these analysis, only 10 sequences were selected and submitted to the prediction of B cell epitope using BCpred tool (Fig. 1). Based on the presence of predicted B cell epitopes and also on the presence of this B cells epitopes, only in S. mansoni protein or in $S$. mansoni protein region without identity with-human proteins, six candidates were selected (Table): a $200 \mathrm{kDa}$ glycophosphatidylinositol (GPI)-anchored surface glycoprotein (Smp_017730), an expressed protein of $12.8 \mathrm{kDa}$ (Smp_034420.1), a cytochrome oxidase subunit, putative of $16 . \overline{5} \mathrm{kDa}$ (Smp_184550), a cytochrome oxidase subunit, putative of $18.9 \mathrm{kDa}$ (Smp_184440), a hypothetical protein of $127.8 \mathrm{kDa}(\mathrm{Smp} 171300)$ and an expressed protein of $43.5 \mathrm{kDa}$ (Smp_042910). Predicted B cell epitopes with scores higher than 0.9 are demonstrated in the protein sequence (Fig. 2). In order to characterize these six selected proteins we performed in silico analysis to predict molecular weight, isoelectric point and glycosylation site. Fig. 3 shows the signal peptide position in the protein sequence as well as predicted $\mathrm{O}$-glycosylation sites.

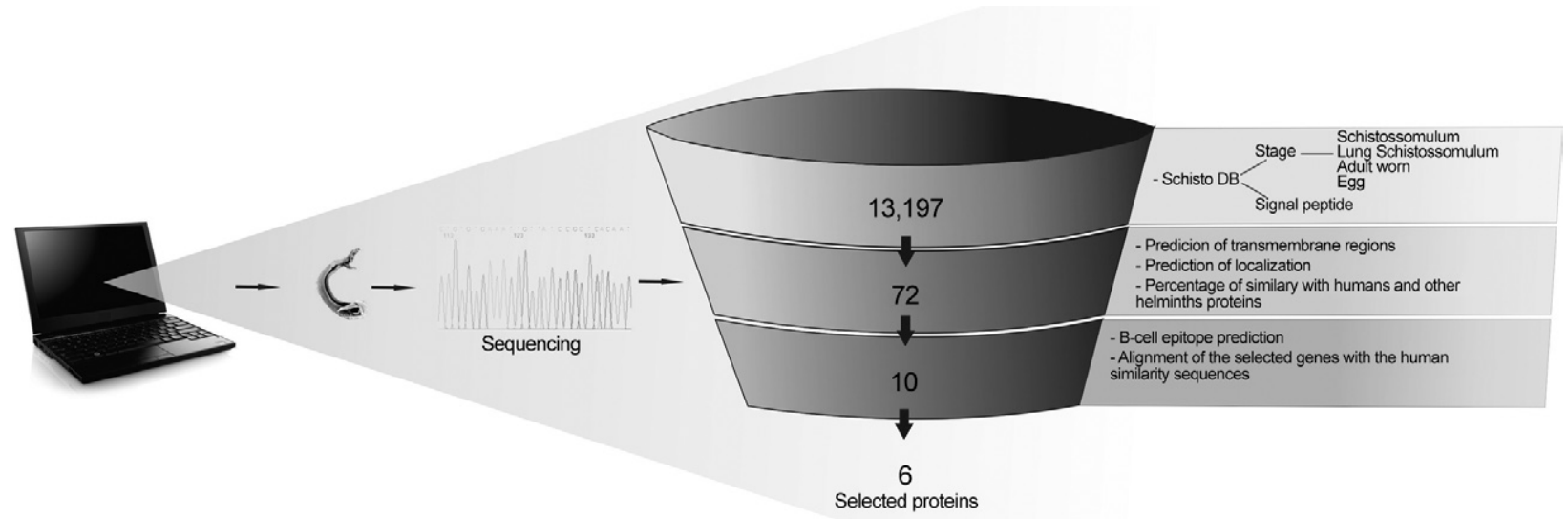

Fig. 1: computational analysis for selection of potential antigens for immunodiagnosis. From genome sequence information, potential antigens were identified using bioinformatics tools. The first analysis was performed in 13.197 schistosome transcripts using SchistoDB (schistodb.net/ schistodb20/) database. Based on evidence of gene expression at different stages of the life cycle of the parasite on the definitive host and prediction of signal peptide, this number was reduced to 72 sequences. The prediction of transmembrane domain, cellular localization and percentage of similarity with human and helminthic proteins led to the selection of 10 proteins. B-cell epitope prediction analysis resulted in selection of six candidates to be used in serological diagnosis of the disease. Adapted from Ulmer et al. (2006). 


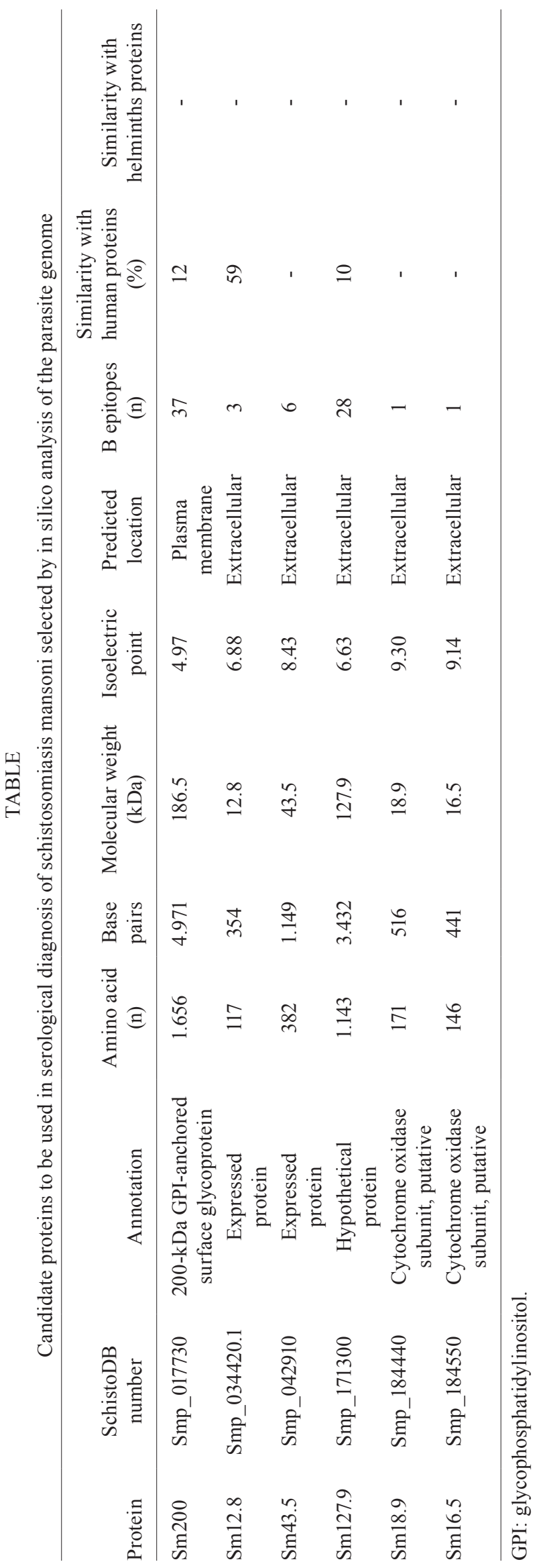

Western blot $1 D$ - In order to evaluate the immunogenicity of the proteins selected based on our filter criteria, a 1D Western blot was performed using adult worm and schistosomula preparation and sera from mice infected with S. mansoni. In Fig. 4A, line 3, a recognition of proteins with approximately $200 \mathrm{kDa}, 100 \mathrm{kDa}$, $43 \mathrm{kDa}$ and $18 \mathrm{kDa}$ by sera from infected mice in AWP was observed. Also in Fig. 4A, line 5, a similar recognition pattern of proteins from SAP with approximately molecular weight of $100 \mathrm{kDa}, 43 \mathrm{kDa}$ and $18 \mathrm{kDa}$ by sera of infected mice could be observed. The recognition observed in lines 3 and 5 is due to $S$. mansoni infection since sera from non-infected mice were unable to recognize these proteins (lines 4,6 ). In order to evaluate if sera from infected mice are able to recognize proteins with lower molecular weight, a Western blot was performed in 12\% SDS-PAGE using SAP. In Fig. 4B, line 2, the recognition of a protein with approximately $14.4 \mathrm{kDa}$ could be observed. Also in this Western blot the recognition of proteins with approximately molecular weight of $18 \mathrm{kDa}$ and $43 \mathrm{kDa}$ could be observed.

Western blot $2 D$ - To further characterize the proteins from AWP recognized by sera from infected mice, a Western blot in a 2D gel was performed. As shown in Fig. 5, many spots from AWP were recognized by sera from infected mice. The spots inside the circles may correspond to the antigens selected by the in silico selection strategy, since they have an isoelectric point and molecular weight similar to the ones predicted to each protein. Spots corresponding to proteins of molecular weight higher than $82 \mathrm{kDa}$ could not be observed in this $2 \mathrm{D}$ Western blot (Fig. 5).

\section{DISCUSSION}

Due to decades of control program based on chemotherapy, the schistosomiasis epidemiological profile in Brazil has changed, with an increase in the number of low schistosome endemic areas (Katz 1998). The low excretion of eggs in faeces makes the diagnosis difficult when the Kato-Katz method is used, there is thus a need for a more sensitive test. Immunodiagnosis based on the presence of antibodies increases the detection of positive cases and constitutes especially a useful method for schistosomiasis diagnosis (Sorgho et al. 2005, Alarcón de Noya et al. 2007). Parasite antigens preparations are examples of antigens used for schistosomiasis diagnosis based on ELISA. A sensitivity of $94-100 \%$ (Ishida et al. 2003, Sorgho et al. 2005) or 96-100\% (Sorgho et al. 2005, Alarcón de Noya et al. 2007) can be observed when adult worm or egg antigens are used in this serological assay, respectively. However, cross reactivity between schistosomiasis and other helminthoses has been reported (Ishida et al. 2003).

A more rational strategy to select candidates for schistosomiasis serological diagnosis can be now performed using bioinformatic tools and the genome information available for S. mansoni (Berriman et al. 2009). Herein, we performed bioinformatics analysis of $S$. mansoni genome in order to identify promising antigens to be used in a diagnostic assay for schistosomiasis. The first analysis was based on the evidence of gene expres- 
sion in all life cycle forms inside the definitive host and also in the presence of signal peptides. These two inclusion criteria reduced the 13.197 transcript described for S. mansoni (Berriman et al. 2009) to 72 protein sequences, representing $0.54 \%$ of the parasite transcriptome. These proteins have a potential to induce an antibody response during infection since they were predicted to be expressed and also may be exposed to host immune system. Signal peptides can drive proteins to a secretion pathway, to membrane and also to intracellular compartment (Blobel 1980). In order to verify if these 72 proteins are secreted or localized in the parasite membrane, we performed a transmembrane domain and cellular localization predictions. The result demonstrates that

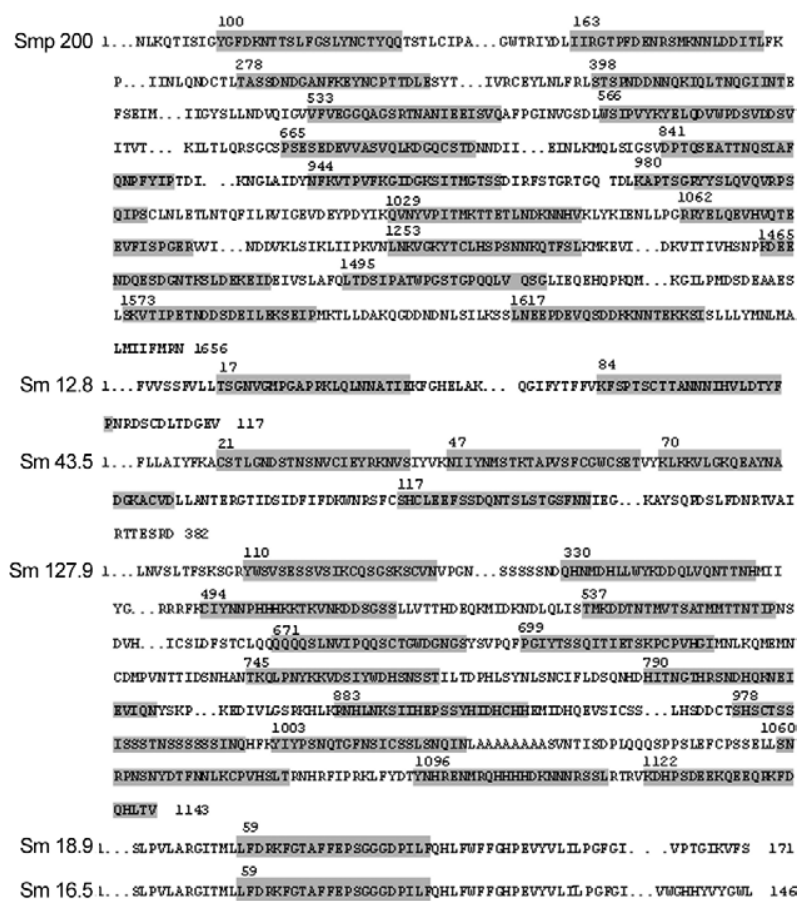

Fig. 2: B-cell epitope in the selected proteins. The highlighted amino acids within the protein sequence represent $\mathrm{B}$-cell epitopes predicted using BCpreds and its amino acid position in the protein sequence.

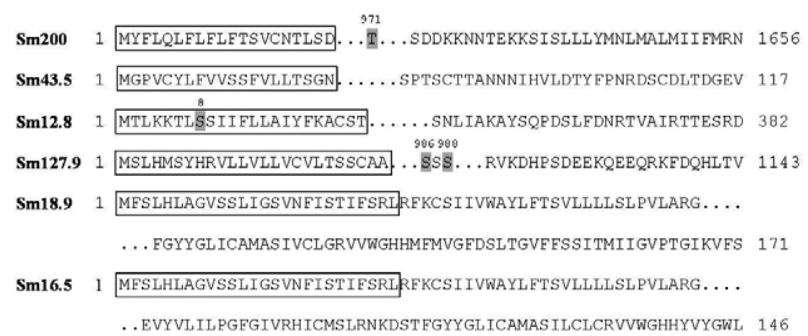

Fig. 3: signal peptide and glycosylation site in selected proteins. The square represents the predicted peptide signal using SignalP and the highlighted amino acid represents the site for predicted glycosylation using OGPET v 1.0 algorithm. The amino acid position for glycosylation sites are pointed in the protein sequence.
59 sequences had at least one transmembrane domain. Cellular localization prediction indicated that only 20 sequences corresponded to transmembrane proteins and 16 sequences corresponded to secreted proteins. The difference in transmembrane domain and cellular localization predictions could be explained by the way these algorithms analyze amino acid sequences. SOSUI identifies transmembrane region based on hydrophobicity of the sequence (Hirokawa et al. 1998) whereas Sherloc
A

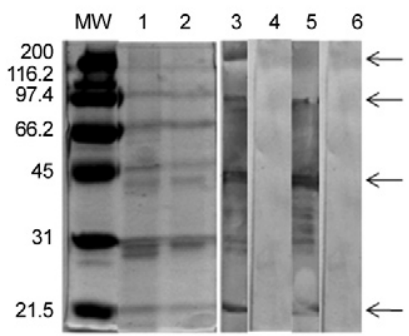

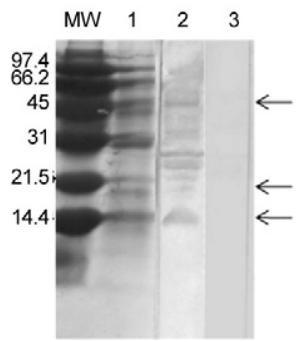

Fig. 4: recognition of schistosomula and adult worm proteins by sera from mice infected with Schistosoma mansoni. Ten micrograms of schistosomula (Lines 1, 3, 4 from A, 1-3 from B) and adult worm proteins (Lines 2, 5, 6 from A) were separated in a $10 \%$ (A) or $12 \%$ (B) sodium dodecyl sulfate polyacrylamide gel electrophoresis and transferred to a nitrocellulose membrane by Western blot. Afterwards, the membranes were probed with sera from mice infected with $S$. mansoni (in A, Lines 3, 5, in B, 2) or with sera from non-infected mice as a negative control (in A, Lines 4, 6, in B, 3). Molecular weight (MW), Lines 1, 2 (A) and 1 (B) were stained with comassie blue.

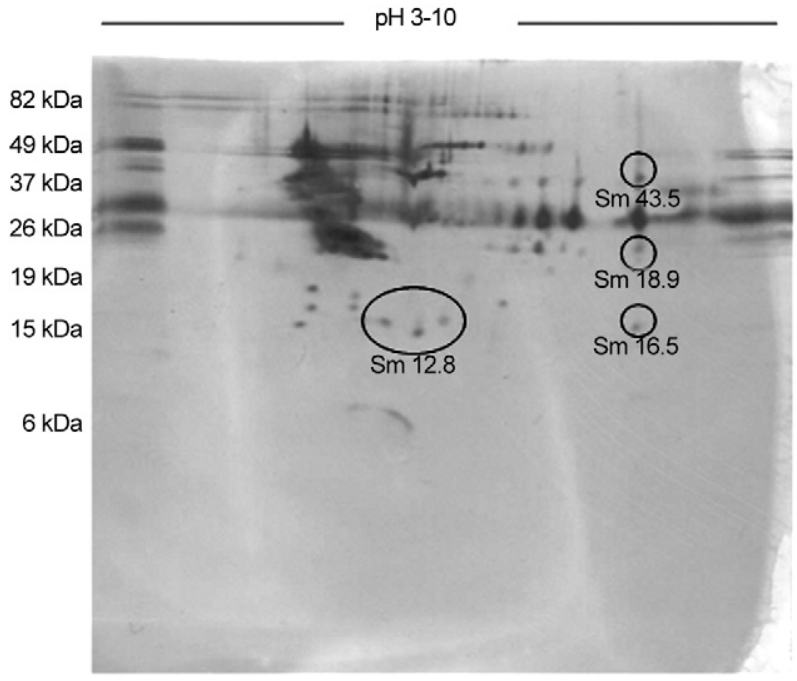

Fig. 5: molecular weight and isoelectric profile from proteins recognized by Schistosoma mansoni infected mice sera. One hundred micrograms of adult worm antigens were separated in a bidimensional gel using immobilized $\mathrm{pH}$ gradient strip $\mathrm{pH} 3-10$ and $15 \%$ sodium dodecyl sulfate polyacrylamide gel electrophoresis. The proteins were transferred to a polyvinylidene difluoride membrane and probed with sera from mice infected with $S$. mansoni. The circles demonstrate the spots that probably represent the antigens selected by our in silico analyzes. 
predicts subcellular location based on a set of features that correlate with location (Shatkay et al. 2007).

Another important characteristic to be considered while analysing genome search for candidates to be used in diagnostic assays is its similarity to human proteins, since auto reactive clones are eliminated or regulated by the immune system (Abbas et al. 1997) leading to poor immunogenic antigens. Our results demonstrated that in the 72 amino acids sequences, 59 had similarity with human proteins ranging from $9-99 \%$. High similarity with human proteins was also an exclusion criterion. Proteins with similarity with human proteins higher than $60 \%$ were excluded from our screening.

Cross reactivity of schistosoma antigens with other helminthic antigens is also a concern while screening candidates for immunodiagnosis. S. mansoni and soil transmitted helminths distribution usually coincides (Raso et al. 2006, Brooker \& Clements 2009, Clements et al. 2010). Even the use of a protein from the parasite as antigens in ELISA instead of parasite extract may result in cross recognition. Recently, the recombinant forms of Sj14-3-3 and Sj26-GST were used for Schistosoma japonicum diagnosis with $94.4 \%$ and $80.7 \%$ sensibility, respectively, but with cross-reaction with Clonorchis sinensis and hookworms (Luo et al. 2009). In our screening selection, similarity with other helminthic proteins was one of the exclusion criteria. Nevertheless, none of the 72 sequences selected in the first analysis had any similarity with other helminthic protein besides schistosome. Considering the cellular location and similarity to human protein, 10 proteins out of the 72 sequences analysed were selected. In order to predict immunogenicity, B cell epitope prediction was performed. All proteins had B cell epitopes predicted in their sequences, the ones that presented B cell epitopes predicted in the region of similarity between $S$. mansoni and human proteins were excluded from our screening. At the end of all analysis we selected six candidates to be used in serological diagnosis for schistosomiasis with predicted molecular weight ranging from 12.8-186.5, and predicted isoelectric point ranging from 4.97-9.3. One of this protein sequence corresponded to Sm200, a GPI-anchored protein present in S. mansoni tegument (Braschi et al. 2006, Castro-Borges et al. 2011) that serves as a target for antibodies and seems to contribute to praziquantel therapeutic action (Sauma et al. 1991). Sm12.8 and Sm43.5 were annotated as expressed proteins; Sm127.9 is a hypothetical protein. Two others selected proteins, Sm16.5 and $\mathrm{Sm} 18.9$, are putative cytochrome oxidase subunits, a protein involved in glucose metabolism that is expressed in cercaria tail and male and female adult worm (Skelly et al. 1993). Although Sm16.5 and Sm18.9 were selected in our screening strategy, they may not represent good candidates to be used on schistosomiasis diagnosis, since cytochrome oxidase probably will not be available to host immune system recognition thus not inducing antibodies production in an infected individual.

The immunogenicity of schistosome proteins were accessed in a 1D and 2D Western blot, using schistosomula antigen preparation, adult worm preparation and sera from infected mice. Antibodies from the sera of infected mice recognize many proteins in the parasite preparation, some of the recognized proteins have molecular weight and isoelectric point similar to those predicted or documented for the proteins. An exception could be observed in Sm12.8 and Sm127.9 that may have had a different migration pattern due to post-translational modification. Although the identification of protein spots recognized by sera from infected mice through mass spectrometry is necessary, the Western blot 1D and $2 \mathrm{D}$ results indicate a good correlation between these two approaches and our in silico screening strategy in selecting immunogenic proteins.

\section{ACKNOWLEDGEMENTS}

To the Program for Technological Development in Tools for Health-Fiocruz, for use of its facilities.

\section{REFERENCES}

Abbas AK, Lichtman AH, Poder JS 1997. Self-tolerance and autoimmunity. In AK Abbas, AH Lichtman, JS Poder, Cellular and molecular immunology, chapter 19, WB Saunders Company, Philadelphia, p. 406-422.

Alarcón de Noya B, Ruiz R, Losada S, Colmenares C, Contreras R, Cesari IM, Noya O 2007. Detection of schistosomiasis cases in low-transmission areas based on coprologic and serologic criteria the Venezuelan experience. Acta Trop 103: 41-49.

Bai X, Borrow R 2010. Genetic shifts of Neisseria meningitidis serogroup $\mathrm{B}$ antigens and the quest for a broadly cross-protective vaccine. Expert Rev Vaccines 9: 1203-1217.

Barbosa CS, Montenegro SML, Abat FGC, Domingues ALC 2001. Specific situations related to acute schistosomiasis in Pernambuco, Brazil. Mem Inst Oswaldo Cruz 96 (Suppl.): 169-172.

Berriman M, Haas BJ, LoVerde PT, Wilson RA, Dillon GP, Cerqueira GC, Mashiyama ST, Al-Lazikani B, Andrade LF, Ashton PD, Aslett MA, Bartholomeu DC, Blandin G, Caffrey CR, Coghlan A, Coulson R, Day TA, Delcher A, DeMarco R, Djikeng A, Eyre T, Gamble JA, Ghedin E, Gu Y, Hertz-Fowler C, Hirai H, Hirai Y, Houston R, Ivens A, Johnston DA, Lacerda D, Macedo CD, McVeigh P, Ning Z, Oliveira G, Overington JP, Parkhill J, Pertea M, Pierce RJ, Protasio AV, Quail M A, Rajandream MA, Rogers J, Sajid M, Salzberg SL, Stanke M, Tivey AR, White O, Williams DL, Wortman J, Wu W, Zamanian M, Zerlotini A, Fraser-Liggett C M, Barrell BG, El-Sayed NM 2009. The genome of the blood fluke Schistosoma mansoni. Nature 460: 352-358.

Blobel G 1980. Intracellular protein topogenesis. Proc Natl Acad Sci USA 77: 1496-1500.

Bradford MM 1976. A rapid and sensitive method for the quantitation of microgram quantities of protein utilizing the principle of protein-dye binding. Anal Biochem 72: 248-254.

Braschi S, Borges WC, Wilson RA 2006. Proteomic analysis of the schistosome tegument and its surface membranes. Mem Inst Oswaldo Cruz 101 (Suppl. I): 205-212.

Brooker S, Clements AC 2009. Spatial heterogeneity of parasite coinfection: determinants and geostatistical prediction at regional scales. Int J Parasitol 39: 591-597.

Capecchi B, Serruto D, Adu-Bobie J, Rappuoli R, Pizza M 2004. The genome revolution in vaccine research. Curr Issues Mol Biol 6: 17-28.

Castro-Borges W, Dowle A, Curwen RS, Thomas-Oates J, Wilson RA 2011. Enzymatic shaving of the tegument surface of live 
schistosomes for proteomic analysis: a rational approach to select vaccine candidates. PLoS Negl Trop Dis 5: e993.

Clements AC, Deville MA, Ndayishimiye O, Brooker S, Fenwick A 2010. Spatial co-distribution of neglected tropical diseases in the east African great lakes region: revisiting the justification for integrated control. Trop Med Int Health 15: 198-207.

Correa-Oliveira R, Dusse LM, Viana IR, Colley DG, Santos Carvalho O, Gazzinelli G 1988. Human antibody responses against schistosomal antigens. I. Antibodies from patients with Ancylostoma, Ascaris lumbricoides or Schistosoma mansoni infections react with schistosome antigens. Am J Trop Med Hyg 38: 348-355.

Enk MJ, Amorim A, Schall VT 2003. Acute schistosomiasis outbreak in the metropolitan area of Belo Horizonte, Minas Gerais: alert about the risk of unnoticed transmission increased by growing rural tourism. Mem Inst Oswaldo Cruz 98: 745-750.

Enk MJ, Lima AC, Massara CL, Coelho PM, Schall VT 2008. A combined strategy to improve the control of Schistosoma mansoni in areas of low prevalence in Brazil. Am J Trop Med Hyg 78: 140-146.

Feldmeier H, Poggensee G 1993. Diagnostic techniques in schistosomiasis control. A review. Acta Trop 52: 205-220.

Hirokawa T, Boon-Chieng S, Mitaku S 1998. SOSUI: classification and secondary structure prediction system for membrane proteins. Bioinformatics 14: 378-379.

Ishida MM, Rubinsky-Elefant G, Ferreira AW, Hoshino-Shimizu S, Vaz A J 2003. Helminth antigens (Taenia solium, Taenia crassiceps, Toxocara canis, Schistosoma mansoni and Echinococcus granulosus) and cross-reactivities in human infections and immunized animals. Acta Trop 89: 73-84.

Kato-Hayashi N, Kirinoki M, Iwamura Y, Kanazawa T, Kitikoon V, Matsuda H, Chigusa Y 2010. Identification and differentiation of human schistosomes by chain reaction. Experimental Parasitology 124: 325-329.

Katz N 1998. Schistosomiasis control in Brazil. Mem Inst Oswaldo Cruz 93 (Suppl. I): 33-35.

Kongs A, Marks G, Verle P, Van det Stuyft P 2001. The unreliability of the Kato-Katz technique limits its usefulness for evaluation of S. mansoni infections. Trop Med Int Health 6: 163-169.

Laemmli UK 1970. Cleavage of structural proteins during the assembly of the head of bacteriophage T4. Nature 227: 680-685.
Luo QL, Qiao ZP, Zhou YD, Li XY, Zhong ZR, Yu YJ, Zhang SH, Liu M, Zheng MJ, Bian MH, Shen JL 2009. Application of signaling protein 14-3-3 and $26 \mathrm{kDa}$ glutathione-S-transferase to serological diagnosis of schistosomiasis japonica. Acta Trop 112: 91-96.

Ramalho-Pinto FJ, Gazzinelli G, Howells RE, Mota-Santos TA, Figueiredo EA, Pellegrino J 1974. Schistosoma mansoni: defined system for stepwise transformation of cercaria to schistosomule in vitro. Exp Parasitol 36: 360-372.

Raso G, Vounatsou P, Singer BH, N'Goran EK, Tanner M, Utzinger J 2006. An integrated approach for risk profiling and spatial prediction of Schistosoma mansoni-hookworm coinfection. Proc Natl Acad Sci USA 103: 6934-6939.

Sauma SY, Tanaka TM, Strand 1991. Selective release of a glycosylphosphatidylinositol-anchored antigen from the surface of Schistosoma mansoni. Mol Biochem Parasitol 46: 73-80.

Shatkay H, Höglund A, Brady S, Blum T, Dönnes P, Kohlbacher O 2007. SherLoc: high-accuracy prediction of protein subcellular localization by integrating text and protein sequence data. Bioinformatics 23: 1410-1417.

Skelly PJ, Stein LD, Shoemaker CB 1993. Expression of Schistosoma mansoni genes involved in anaerobic and oxidative glucose metabolism during the cercaria to adult transformation. Mol Biochem Parasitol 60: 93-104.

Sorgho H, Bahgat M, Poda JN, Song W, Kirsten C, Doenhoff MJ, Zongo I, Ouédrago J B, Ruppel A 2005. Serodiagnosis of Schistosoma mansoni infections in an endemic area of Burkina Faso: performance of several immunological tests with different parasite antigens. Acta Trop 93: 169-180.

Towbin H, Staehelin T, Gordon J 1979. Electrophoretic transfer of proteins from polyacrylamide gels to nitrocellulose sheets: procedure and some applications. Proc Natl Acad Sci USA 76: $4350-4354$.

Ulmer JB, Wahren B, Liu MA 2006. Gene-based vaccines: recent technical and clinical advances. Trends Mol Med 12: 216-222.

Zhao BP, Chen L, Zhang YL, Yang JM, Jia K, Sui CY, Yuan CX, Lin JJ, Feng XG 2011. In silico prediction of binding of promiscuous peptides to multiple MHC class-II molecules identifies the Th1 cell epitopes from secreted and transmembrane proteins of Schistosoma japonicum in BALB/c mice. Microbes Infect 13: 709-719. 